Research Paper

\title{
Topical Application of Tat-Racl Promotes Cutaneous Wound Healing in Normal and Diabetic Mice
}

\author{
Bin Fan ${ }^{1,5^{*}}$, Tao Wang ${ }^{1,6^{*}}$, Li Bian ${ }^{1,7^{*}}$, Zhe Jian ${ }^{1,8}$, Dongyan D. Wang1, Fulun Li1,5, Fanglong Wu1 ${ }^{1}$, Tao Bai ${ }^{1}$, \\ Gongyi Zhang'2, Nik Muller³, Barry Holwerda ${ }^{4}$, Gangwen Han ${ }^{1,9 * *}$, Xiao-Jing Wang1,10凶 \\ 1. Department of Pathology, University of Colorado Denver Anschutz Medical Campus, Aurora, CO, USA. \\ 2. Department of Biomedical Research, National Jewish Health, CO, USA. \\ 3. Department of Ophthalmology, University of Colorado Denver Anschutz Medical Campus, Aurora, CO, USA. \\ 4. Molecular Throughput Inc., Las Vegas, NV89118, USA. \\ 5. Current address: Department of Dermatology, Yueyang Hospital of Integrated Traditional Chinese and Western Medicine, Shanghai University of \\ Traditional Chinese Medicine, Shanghai, China \\ 6. Current address: Institute of Combined Injury, State Key Laboratory of Trauma, Burn and Combined Injury, Chongqing Engineering Research Center for \\ Nanomedicine, College of Preventive Medicine, Third Military Medical University, Chongqing, China \\ 7. Department of Pathology, the First Affiliated Hospital of Kunming Medical University, Kunming. P.R. China \\ 8. Current address: Department of Dermatology, Xijing Hospital, Fourth Military Medical University, Xi'an, Shanxi, China \\ 9. Current address: Department of Dermatology, Peking University International Hospital, Beijing 102206, China \\ 10. Veterans Affairs Medical Center, VA Eastern Colorado Health Care System, Aurora, CO, USA
}

*The authors contributed equally to this work.

$\triangle$ Corresponding author: Gangwen Han, Department of Dermatology, Peking University International Hospital, Life Park Road No.1, Life Science Park of Zhong Guancun, Changping District, Beijing 102206, China. Phone: (+86 10) 6900 6205; E-mail: hangangwen@pkuih.edu.cn; Xiao-Jing Wang, Department of Pathology, University of Colorado Denver Anschutz Medical Campus, Aurora, CO, USA, E-mail: XJ.Wang@ucdenver.edu

(c) Ivyspring International Publisher. This is an open access article distributed under the terms of the Creative Commons Attribution (CC BY-NC) license (https://creativecommons.org/licenses/by-nc/4.0/). See http://ivyspring.com/terms for full terms and conditions.

Received: 2018.03.06; Accepted: 2018.06.09; Published: 2018.06.23

\begin{abstract}
The endogenous small GTPase, Racl, plays a critical role during normal skin wound healing. It remains to be determined whether endogenous Racl can be appropriately activated in chronic wounds; if not, whether exogenous Racl has therapeutic effects on wound healing. Here we show that Racl protein levels were lower in wounds of $\mathrm{db} / \mathrm{db}$ diabetic mice than wounds in wild type mice during the healing process. To assess the therapeutic potential of exogenous Racl in wound healing, we produced a Tat-Racl fusion protein that enters into cells through protein transduction. Tat-Racl increased proliferation and migration of keratinocytes and dermal fibroblasts in vitro. Topical application of Tat-Racl accelerated cutaneous wound closure in vivo in $\mathrm{db} / \mathrm{db}$ mice as well as wild type mice. Further analyses revealed that Tat-Racl had faster re-epithelialization, higher keratinocyte proliferation and migration without an earlier onset of myofibroblast activation than vehicle treated wounds. Tat-Racl also reduced inflammation in wounds. Our findings revealed the failure of diabetic wounds to elevate Racl expression and suggested a therapeutic strategy utilizing a Racl-based biologic to compensate for this defect thereby promoting wound healing.
\end{abstract}

Key words: Rac1; Tat-Rac1; wound healing; diabetic wounds

\section{Introduction}

Chronic skin wounds including pressure ulcers, diabetic foot ulcers and venous ulcers are serious medical problems worldwide. It has been reported those diseases affect 6.5 million patients in the United States alone. Together with aberrant healing in acute skin wounds (e.g., infection and scarring), the burden to treat these conditions exceeds $\$ 25$ billion annually [1]. Strategies developing innovative targets to accelerate wound healing are based on the current mechanistic understanding of cutaneous wound healing. Cutaneous wound healing progresses through three distinct, yet overlapping phases: inflammation, tissue formation, and tissue remodeling [2]. Each phase is complex and involves coordinated interactions among tissue repair cells, growth factors and extracellular matrix [3]. Numerous 
growth factors and cytokines have been shown to contribute to wound healing [3]. However, among them, only REGRANEX ${ }^{\circledR}$, a human PDGF-based therapeutic gel, is approved by the Food and Drug Administration (FDA) to treat diabetic wounds. These facts highlight a sense of urgency for scientific discovery-based therapeutic interventions. Various studies have identified Rac1, a small GTPase, as playing an important role in wound healing. Deletion or inhibition of Rac1 in either skin epidermis or oral epithelia resulted in delayed wound healing in the skin or the oral mucosa [4,5]. Similarly, inducible deletion of the Rac1 gene in fibroblasts resulted in delayed wound closure with reduced collagen production and myofibroblast formation [6]. Conversely, transgenic mice expressing a constitutively active Rac1 in smooth muscle cells accelerate cutaneous wound repair through promoting angiogenesis [7]. In addition to its role in normal wound healing, a study reported that ganglioside GM3 depletion in diabetic mouse wounds improved healing by requiring IGF-1 receptor-mediated Rac1 activation to restore keratinocyte motility [8, 9]. Additionally, ultrasonic treatment-mediated promotion of wound healing in mouse models of diabetic and aging skin wounds involves activation of the calcium/CamKinaseII/Tiam1/Rac1 pathway that promotes fibroblast migration[10]. These data suggest that impaired wound healing lacks sufficient Rac1 activity and that activation of endogenous Rac1 should be explored as a therapeutic strategy to improve wound healing. In the present study, we sought to determine if Rac1 protein can be utilized pharmacologically to promote wound healing. Because Rac1 is an intracellular protein, it has to be delivered to the inside of cells to exert its functions. To this end, we employed a protein transduction strategy to deliver Rac1 into wounded cells. We chose a protein transduction peptide derived from the Tat protein of the HIV virus, and fused it to human Rac1 to create a Tat-Rac1 protein. Our study provides proof of principle for using Tat-Rac1 as a pharmacological biologic to promote cutaneous wound healing.

\section{Materials and methods}

\section{Reagents and antibodies}

Antibodies used in the present study were: guinea pig anti-K14 (RDI-Fitzgerald, Acton, MA), mouse anti-Rac1, rat anti-CD45, anti-BrdU FITC (BD Bioscience, San Jose, CA), mouse anti-V5 (Invitrogen, Grand Island, NY), mouse anti-GAPDH (Cell Signaling Technology, Danvers, MA), rabbit anti- $\alpha$-smooth muscle actin ( $\alpha$ SMA) (ABcam, Cambridge, UK), and rabbit anti-actin (Santa Cruz
Biotechnology). For immunofluorescence (IF), Alexa Fluor 594 (red) or 488 (green) conjugated secondary antibodies (Invitrogen, Carlsbad, CA) were used. For aSMA immunohistochemistry (IHC), anti-rabbit secondary antibody (Vector Labs, Burlingame, CA) was used. All slides were mounted in Fluoromount $G$ (Southernbio-tech, Birmingham, AL) with coverslips before microscopic examination.

\section{Generation of Tat-Racl protein}

Human Rac1 cDNA with 5' nucleotides encoding the Tat-tag (RKKRRQRRR) and 3' nucleotides encoding the V5 epitope (GLPIPNPLLGLDS) was cloned into the pET101TOPO protein expression vector (Invitrogen) upstream of the 6XHis-tag. The protein was produced in BL21 Star ${ }^{\mathrm{TM}}$ (DE3) E. coli and purified with a Ni-Sepharose column. Protein eluted from Ni-column was loaded onto a Sephacryl S200 size exclusion column (SEC) to separate Tat-Rac1 as a monomer. All reagents and buffers used in protein purification are endotoxin-free grade.

\section{In vitro characterization of transduction and biological activity of Tat-Racl protein}

WT primary mouse keratinocytes were isolated from neonatal mouse skin as previously described [11]. To test the biologic activity and ability of Tat-Rac1 to enter into cells, mouse keratinocytes, human HaCaT keratinocytes (from Thermo Fisher Scientific), or human dermal fibroblasts (ATCC, Manassas, VA) were cultured on the 8-well chamber slides for $12 \mathrm{~h}$, then Tat-Rac1 protein $(1 \mu \mathrm{g} / \mathrm{ml})$ or BrdU $(10 \mu \mathrm{M})$ was added to the culture medium and incubated for 3h; slides were fixed in cold methanol for $5 \mathrm{~min}$ and washed in PBS. V5 or BrdU were stained using anti-V5 or anti-BrdU antibodies. Cell proliferation response to Tat-Rac1 protein was evaluated by the percent of BrdU labelled cells in total counted cells. Each experiment was averaged from 8-well chambers; data from 3 separate experiments as mean \pm SD.

\section{In vitro keratinocyte migration assays}

In vitro keratinocyte migration assays were performed as previously reported [12]. Briefly, WT cultured mouse keratinocytes or human HaCaT cells at $100 \%$ confluency in 6-well culture dishes were incubated with mitomycin C, $10 \mu \mathrm{g} / \mathrm{ml}$, (Sigma, St. Louis, MO) for $2 \mathrm{~h}$ to inhibit cell proliferation. A 1.2 mm diameter pipette tip was used to make the wound scratch. Tat-Rac1 $(1.25 \mu \mathrm{g} / \mathrm{ml}$ in mouse keratinocytes, $1.25 \mu \mathrm{g} / \mathrm{ml}$ or $2.5 \mu \mathrm{g} / \mathrm{ml}$ in HaCaT and human dermal fibroblasts), or equal volume vehicle PBS was added to media. Media in mouse keratinocyte culture was changed after $24 \mathrm{~h}$ with the same concentration of 
Tat-Rac1. Cell migration was quantified using ImageJ for the scratched area occupied by migrating cells at different time points specified in Results. Each experiment was averaged from 6-well 3-mm dishes; data from 3 separate experiments as mean $\pm S D$.

\section{Animals and wound healing experiments}

Experiments were performed in accordance with the National Institutes of Health Guidelines for the Care and Use of Laboratory Animals. The wound healing protocol was approved by the Institutional Animal Care and Use Committee at the University of Colorado Denver Anschutz Medical Campus. Hemizygous db/wt mice were purchased from the Jackson Laboratory (www.jax.org) with C57BL/6J background. We cross-bred $\mathrm{db} / \mathrm{wt}$ and $\mathrm{db} /$ wt mice to generate $\mathrm{db} / \mathrm{db}$ mice, and $\mathrm{db} / \mathrm{wt}$ and $w \mathrm{t} / \mathrm{wt}$ littermates were used as wildtype (WT) controls for wound healing experiments. Animals were housed under a 12 hours light/dark cycle in a SPF room with water and food provided ad libitum. For wounding experiments, of 8-10 week old mice were used for generation of skin wounds as previously reported [11]. Briefly, four full-thickness excisional wounds were made on the dorsal skin of anesthetized mice using a sterile 6-mm-diameter dermal punch. Tat-Rac1 [10ul phosphate buffered saline (PBS), doses specified in Results] was applied to each wound daily until day 8 when they are completely covered by scabs; treatment solution was allowed to dry before mice were returned to their cages. After day 10, Tat-Rac1 was topically applied to the gap between the scab and wound periphery to avoid the hard scab barrier. PBS (10 $\mu \mathrm{l} /$ wound) was used as a vehicle control. Wound healing was evaluated by calculating both the wound area and the histological wound width from wound midline under microscopy.

\section{Histology and Immunostaining analysis}

An 8-mm diameter dermal punch symmetrically covering the entire wound was used to collect each wound sample. The whole wound was subjected to formalin fixation and paraffin embedding. Serial sections were cut from wound midline and H\&E stained. The largest cross section of each wound was used for width measurement under microscopy. IF or IHC staining in tissue and cells were performed as previously described [12]. BrdU was quantified as the number of positive cells per $\mathrm{mm}$ migrating epidermis. CD45+ cells were quantified as positive cells per $\mathrm{mm}^{2}$ stromal area directly under migrating epithelium. For quantification of IF images, we counted positive staining cells in four to five images from each wound and averaged them as one sample; 3 samples/group/time point were used for analyses.

\section{Western blot analysis}

Western blots were performed per our previous publication [12]. Briefly, tissue was homogenized in Complete Lysis-M buffer containing protease inhibitor together with phosphatase inhibitors (Roche Applied Science, Indianapolis, IN). Protein lysate was collected by centrifugation and protein concentrations were determined by Bio-Rad protein assay kit (Bio-Rad Laboratories, Hercules, CA). A total of $60 \mathrm{mg}$ protein lysate was loaded into each lane of a 10\% SDS gel. Proteins were transferred to a nitrocellulose membrane, blocked with 5\% non-fat milk and incubated with primary antibodies at $4^{\circ} \mathrm{C}$ overnight on a slow rocker. Primary antibodies used include mouse anti-Rac1 (1:500, BD Bioscience, San Jose, CA) or mouse anti-V5. Internal control antibodies such as mouse anti-GAPDH (1:500, Cell Signaling Technology, Danvers, MA) or rabbit anti-actin (1:500, Santa Cruz Biotechnology) were used. Membranes were incubated with secondary antibodies for one hour at room temperature [IRDye conjugated donkey anti-rabbit IgG or anti-mouse IgG (1:2000, Rockland Immunochemicals Inc., Gilbertsville, PA)]. Odyssey v.1.2 software (LI-COR Biosciences, Lincoln, NE) was used to convert color images to gray scale images.

\section{Statistical Analysis}

The Student's-test was used to compare two groups. The data are presented by mean \pm SD.

\section{Results}

\section{Endogenous Racl expression in $\mathrm{db} / \mathrm{db}$ and WT mice during wound healing}

To examine endogenous Rac1 expression levels during wound healing, we performed excisional wound experiments with a 6-mm biopsy punch in WT and $\mathrm{db} / \mathrm{db}$ mouse skin $(\mathrm{db} / \mathrm{db}$ mice have delayed wound healing), and collected wounds with an 8-mm biopsy punch at different time points to examine Rac1 protein levels by western blot analysis (Fig 1A). Rac1 protein was barely detectable in non-wounded skin in both $\mathrm{WT}$ and $\mathrm{db} / \mathrm{db}$ mice. During wound healing, Rac1 protein levels gradually increased in WT wounds (Figure 1A); in contrast, $\mathrm{db} / \mathrm{db}$ mouse wounds had lower Rac1 protein levels compared to WT wounds for all time points examined. These results suggest that lower levels of Rac1 in $\mathrm{db} / \mathrm{db}$ mice skin wounds might contribute to delayed wound closure when compared to WT mice wounds.

\section{Tat-Racl protein promotes keratinocyte proliferation and migration}

To test if exogenous Rac1 protein can overcome wound healing defects seen in $\mathrm{db} / \mathrm{db}$ mice, we 
generated a Tat-Rac1 fusion protein, in which a protein transduction peptide (Tat) from the Tat protein of the HIV virus was fused with human Rac1 protein, and with a V5 peptide at the C-terminal of the protein to simplify detection of the recombinant protein (Figure 1B). Tat-Rac1 protein was purified using a size exclusion column (SEC) (Figure 1C). To verify its ability to enter keratinocytes, we added Tat-Rac1 protein to cultured mouse keratinocytes; immunofluorescence staining using a V5 antibody shows Tat-Rac1 protein entered into cells (Figure 1D). We then performed in vitro cell proliferation and migration assays with mouse keratinocytes, $\mathrm{HaCaT}$ human keratinocytes and human dermal fibroblasts to determine Tat-Rac1 bioactivities. For proliferation assays, we examined the number of BrdU labeled cells $12 \mathrm{~h}$ after Tat-Rac1 treatment. In mouse keratinocytes, Tat-Rac1 treatment significantly increased the number of BrdU positive staining cells compared to control cells treated with PBS vehicle (Figure 2A, B). Similarly, Tat-Rac1 also increased proliferation in $\mathrm{HaCaT}$ keratinocytes and dermal fibroblasts (Figure 2C, 2D). In cell migration assays, we examined mouse keratinocyte migration in response to Tat-Rac1 treatment (Figure 2E, 2F). By 24 hours after scratch wounding, when control keratinocytes began to migrate into the wound area, more than $50 \%$ of the wound area was covered by migrating cells treated with Tat-Rac1 protein. By 48 hours, Tat-Rac1 treated keratinocytes almost completely covered the scratch wounded area, whereas control keratinocytes only covered $\sim 40 \%$ of the wounded area. We also found that Tat-Rac1 protein had a similar effect on migration in $\mathrm{HaCaT}$ keratinocytes and human dermal fibroblasts at $12 \mathrm{~h}$ (Figure 2G, 2H) and 24h (not shown). These data indicated that Tat-Rac1 was biologically active.
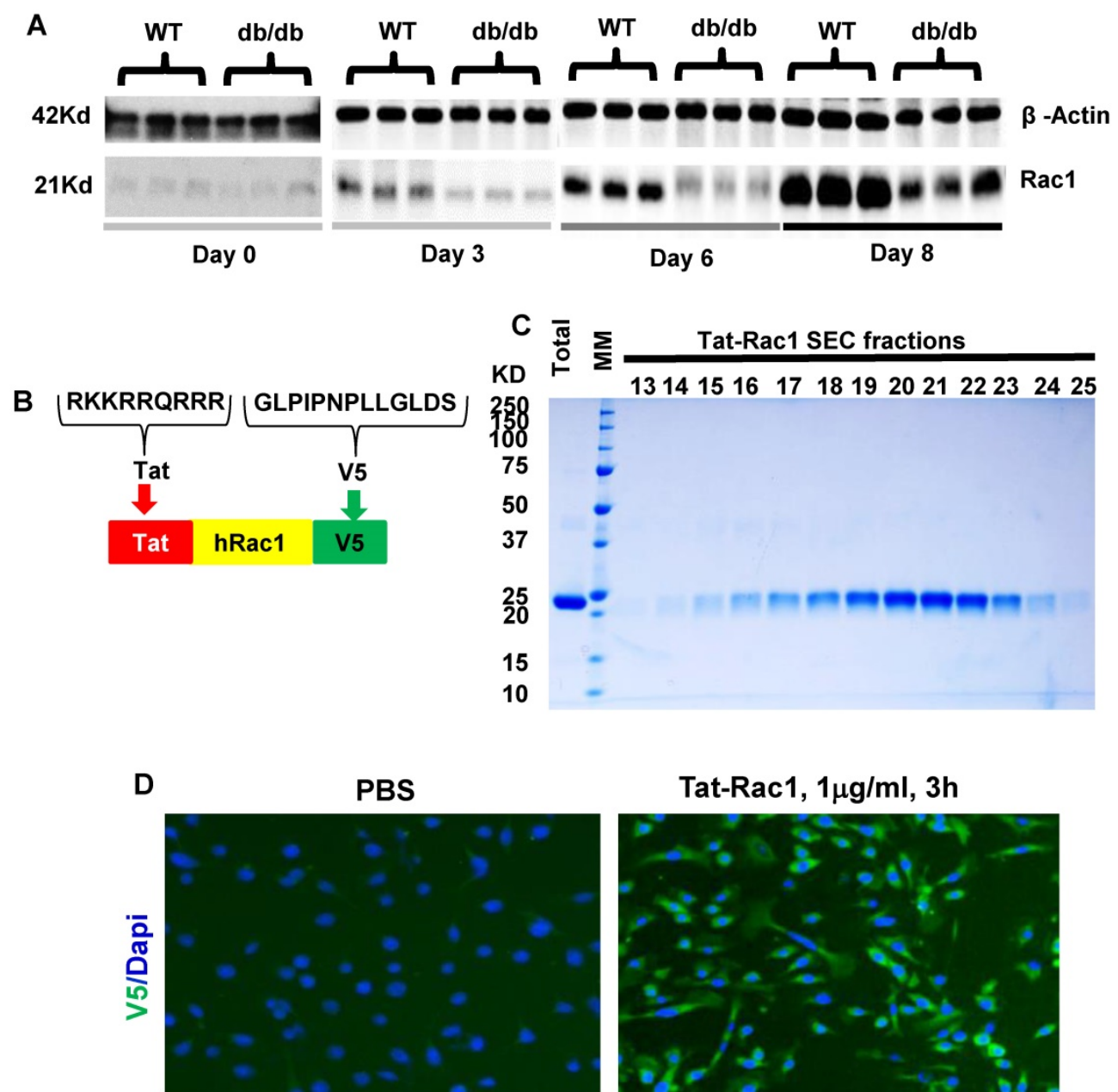

Tat-Rac1, $1 \mu \mathrm{g} / \mathrm{ml}, 3 \mathrm{~h}$

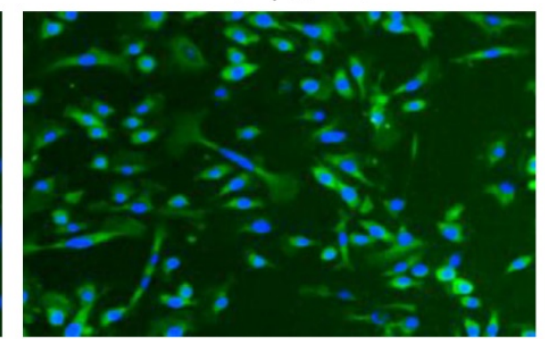

Figure 1. Endogenous Racl protein levels in db/db and WT mouse skin wounds and characterization of Tat-Racl protein. (A) Western blot comparing Racl protein levels between WT and db/db wound wounds at specific time points before (day 0 ) or after wounding. $\beta$-Actin on the same blot was used as a loading control. (B) Schematic showing Tat-Racl protein structure. (C) SDS-PAGE gel image showing total (after cutting GST) and SEC purified Tat-Racl protein (24.2KD). (D) Immunofluorescence staining using V5 antibody showing Tat-Racl entering into cytoplasm of mouse keratinocytes (green), left image, right image negative control. DAPI was used as counterstain to show nuclei. 


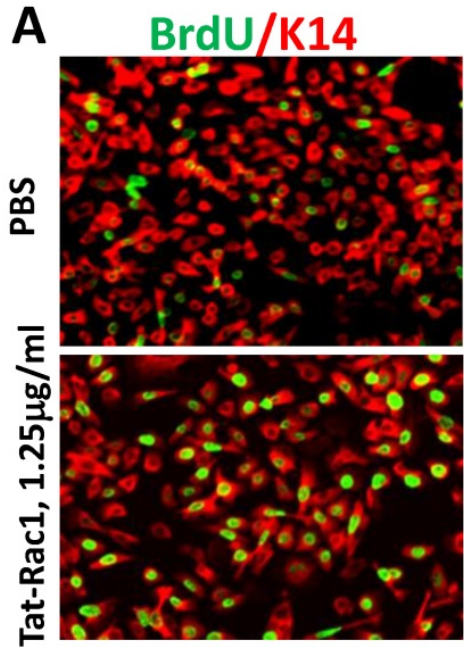

B

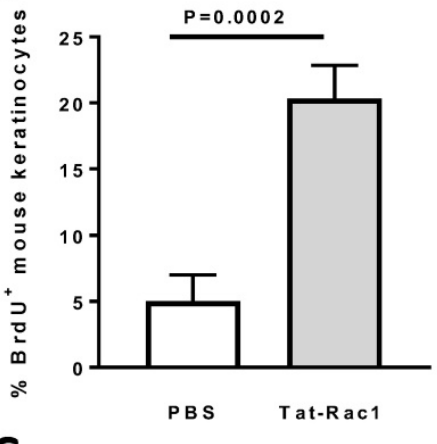

C

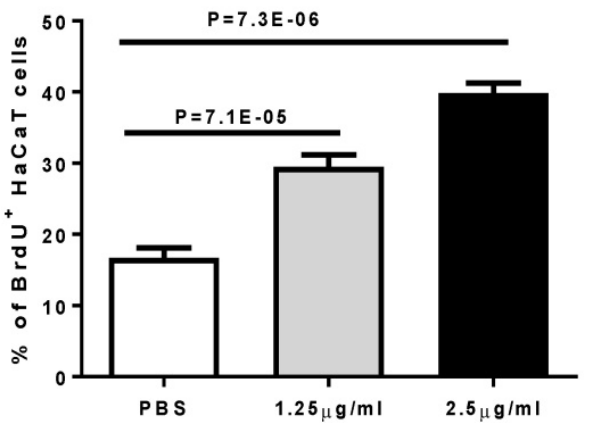

D

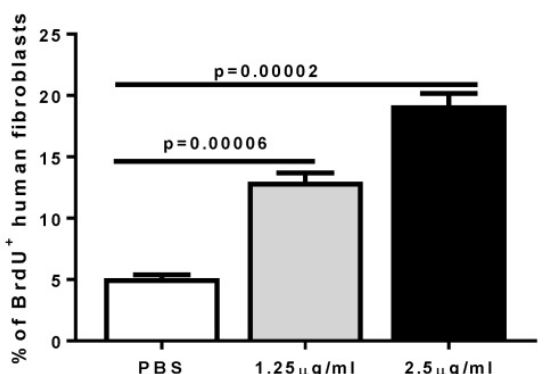

E
PBS

Tat-Rac1, $1.25 \mu \mathrm{g} / \mathrm{ml}$
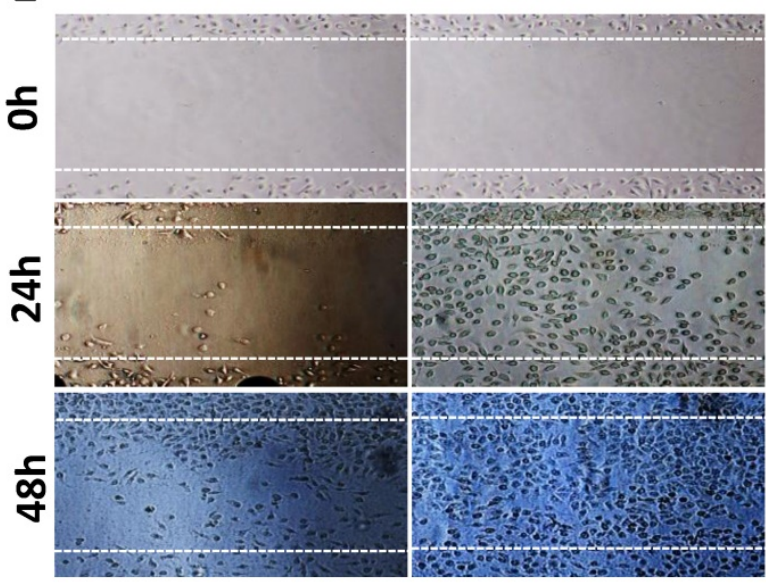

F
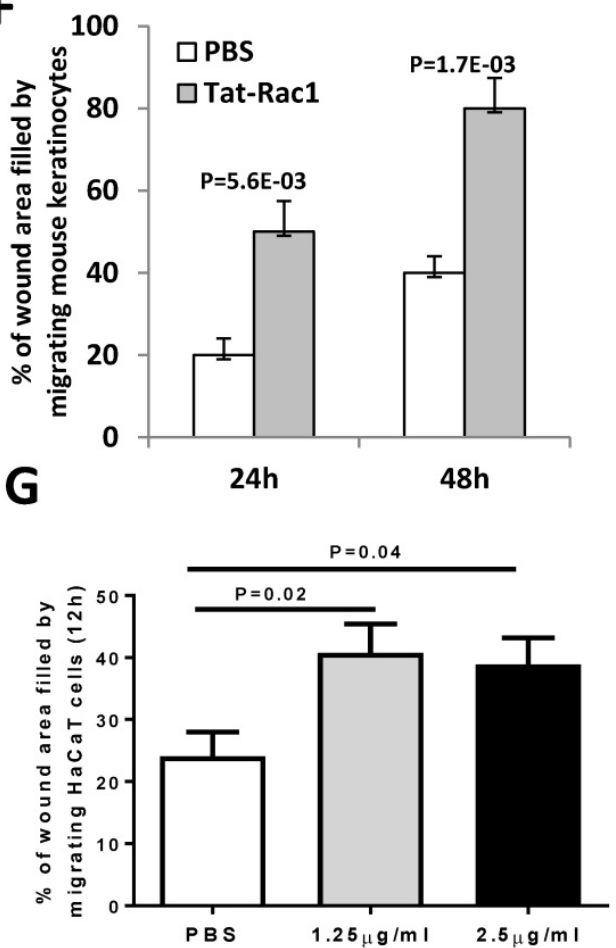

$\mathbf{H}$

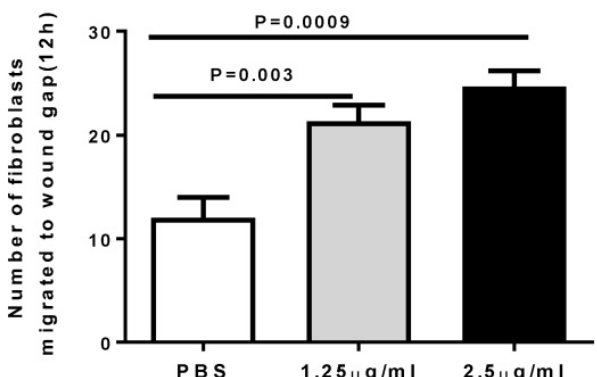

Figure 2. Tat-Racl protein promoted keratinocyte proliferation and migration. (A) Immunofluorescence image of BrdU nuclear staining (green) of WT mouse keratinocytes 12 hours after Tat-Racl treatment. K14 (red) was used as a counterstain. (B-D) Quantification of \% of BrdU positive cells in mouse keratinocytes (B), HaCaT human keratinocytes (C) and human dermal fibroblasts (D), each from 3 separate experiments as mean + SD. Each experiment was averaged from 8 -well chambers. (E) Mouse keratinocyte migration assay with or without Tat-Racl treatment. Dotted lines indicate the wound margin. (F-H) Quantification migrating cells in mouse keratinocytes (F), $\mathrm{HaCaT}$ human keratinocytes $(\mathbf{G})$ and human dermal fibroblasts $(\mathbf{H})$, each from three separate experiments as mean \pm SD. Each experiment was averaged from 6-well chambers. 


\section{Topical application of Tat-Racl protein accelerated cutaneous wound healing in wild type and $\mathrm{db} / \mathrm{db}$ mice}

Next, we examined if topical application of Tat-Rac1 protein can promote wound healing in vivo. Based on clinical practice, diabetic wounds represent a common type of unhealed wound in humans, therefore, we generated wounds in $\mathrm{db} / \mathrm{db}$ mice. Excisional wounds were made with a $6-\mathrm{mm}$ diameter biopsy punch on the dorsal skin of 8-10 wks old $\mathrm{db} / \mathrm{db}$ mice (both males and females). Gendermatched mice were divided into two groups for Tat-Rac1 protein (in10 $\mu$ l PBS) or equal volume of PBS-vehicle treatment, topically applied onto the wound area, starting immediately after punch biopsy (day 1). Based on in vitro experiments (Figure 2) showing the effective dose is likely in the $\mu \mathrm{g}$ range, we tested three doses of Tat-Rac1 (0.5, 1 and $2 \mu \mathrm{g} /$ wound, daily). While $0.5 \mu \mathrm{g} /$ wound had modest effects, $2 \mu \mathrm{g} /$ wound had no effect on promoting healing (not shown). We therefore focused on a1 $\mu \mathrm{g} /$ wound dose for the remaining studies. With Tat-Rac1 treatment, accelerated wound closure was observed beginning on day 4 after wounding compared with PBS treatment (Figure 3A, B). Tat-Rac1-treated $\mathrm{db} / \mathrm{db}$ mice wounds were completely closed by day 13, whereas PBS treated wounds show slower healing for all time points. Histologically, Tat-Rac1 treated wounds were smaller in width and had longer epithelial migrating tongues by day 6 and day 8 compared to PBS controls (Figure 3C, D). On day 13, Tat-Rac1-treated mice had smaller stromal areas in the wound than untreated control mice (Fig 3C). By staining wounded tissue with V5 antibody, Tat-Rac1 protein was detected in epidermal keratinocytes and stromal cells in the wound area (Figure 3E). We also performed western blot analysis using a V5 antibody to confirm the presence of Tat-Rac1 protein on day 8 wounds (Figure 3F).

Next, we tested if Tat-Rac1 application could also provide benefits to normal wound healing. Gender-matched 8-10 week-old WT mice were used for Tat-Rac1 and PBS groups with 6-mm punch skin biopsies. Tat-Rac1 treated wounds exhibited accelerated healing compared to PBS treated wounds starting from day 2 to day 9 (Figure 4A, B). Histological analysis showed that Tat-Rac1 treated wounds had longer epidermal migrating tongues than control wounds on day 5 and day 8 (Figure 4C, D). By day 9, when wound closure was complete in both groups, Tat-Rac1 treated wounds had smaller wound remodeling areas than control group.

\section{Increased keratinocyte proliferation contributed to Tat-Racl-mediated wound healing acceleration}

Due to Tat-Rac1's ability to promote keratinocyte proliferation in vitro (Figure 2), we examined whether topical application of Tat-Rac1 on wounds increased keratinocyte proliferation during wound healing in vivo. Mice were injected with BrdU $2 \mathrm{~h}$ before harvesting wound tissue samples; sections were subsequently stained for BrdU using immunofluorescence. In both $\mathrm{db} / \mathrm{db}$ and WT mice, all Tat-Rac1 treated wounds had higher numbers of BrdU positive cells compared to PBS treated control wounds (Figure $5 \mathrm{~A}-\mathrm{D})$.

\section{Reduced inflammation but not myofibroblast numbers in Tat-Racl treated wound stroma}

Inflammation plays a critical role in the process of wound healing and excessive inflammation is one of the characteristics of chronic wounds. To determine how Tat-Rac1 treatment affects this process, we compared the number of CD45+ leukocytes in $\mathrm{db} / \mathrm{db}$ and WT control wounds with or without Tat-Rac1 treatment. Compared to PBS vehicle treatment, the number of CD45+ cells in wound stroma were decreased in both $\mathrm{db} / \mathrm{db}$ and WT mice after Tat-Rac1 treatment during the early stage of wound healing (Figure 6A-D).

Fibroblast activation is the basis of wound contraction and stromal regeneration, but excessive fibroblast activation can also cause fibrotic scarring. We performed IHC for $\alpha$ SMA, a marker for fibroblast activation and myofibroblast formation. Tat-Rac1 treatment did not induce earlier onset $\alpha$ SMA positive cells or increase $\alpha$ SMA positive cells before day 8 in $\mathrm{db} / \mathrm{db}$ wounds or day 5 in WT wounds (not shown). Cells positive for $\alpha \mathrm{SMA}$ began to appear in granulation tissue (wound stroma) after these time points (Figure $6 \mathrm{E}, 6 \mathrm{~F}$ ). On day $8 \mathrm{db} / \mathrm{db}$ wounds or day 5 WT wounds, $\alpha$ SMA staining was moderately broader or intense in some areas (Figure 6E, 6F). In $\mathrm{db} / \mathrm{db}$ day 13 wounds, even though re-epithelialization was completed in both groups (Figure 6E), aSMA positive cells were retained in granulation tissue, suggesting that wound remodeling was not complete. However, in WT day 9 wounds, where the area of granulation tissue was smaller in Tat-Rac1 treated wound than PBS treated wound, $\alpha$ SMA positive cells had largely disappeared in Tat-Rac1 treated granulation tissue (Figure 6F), suggesting that topically applied Tat-Rac1 does not have a sustained effect on fibroblast activation once the treatment is stopped. 


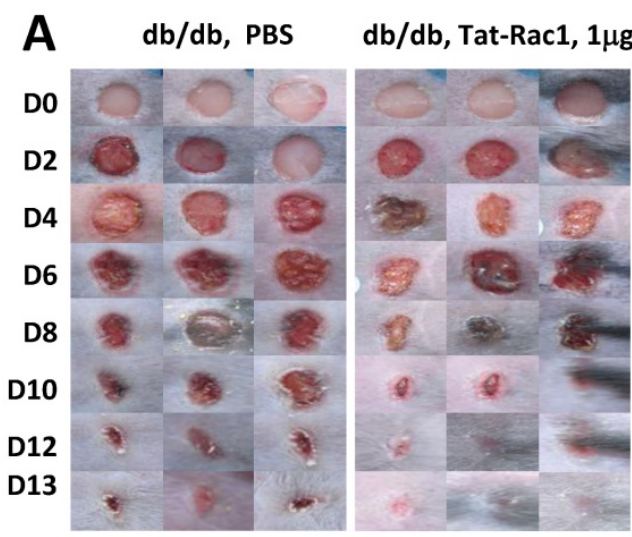

C
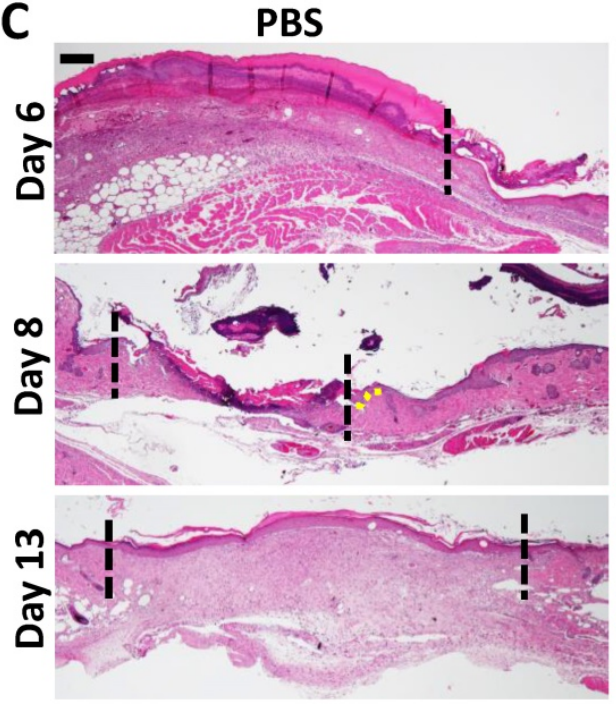

D

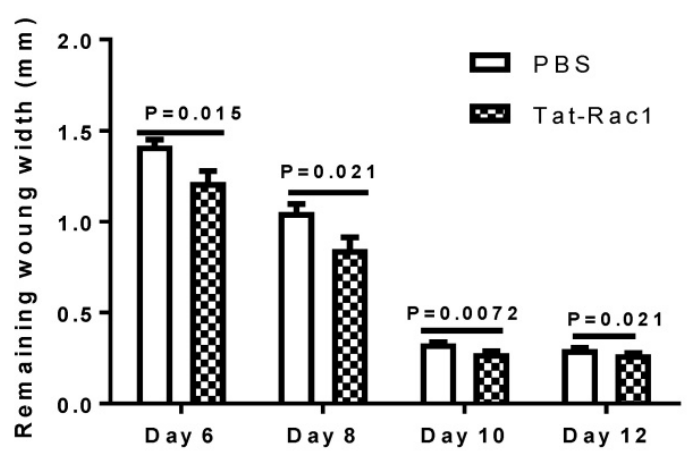

B

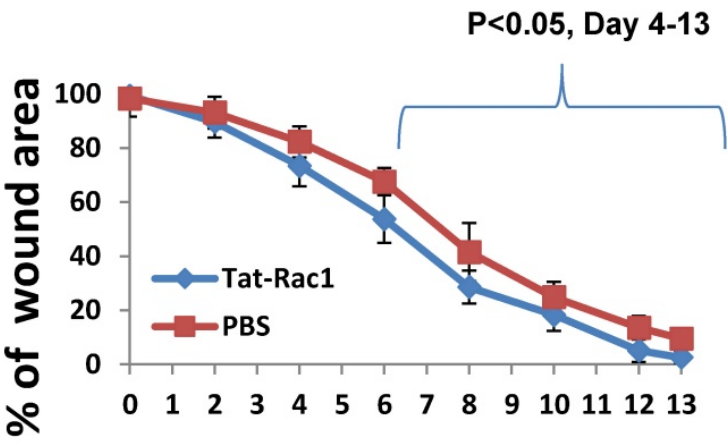

\section{Days after wounding}
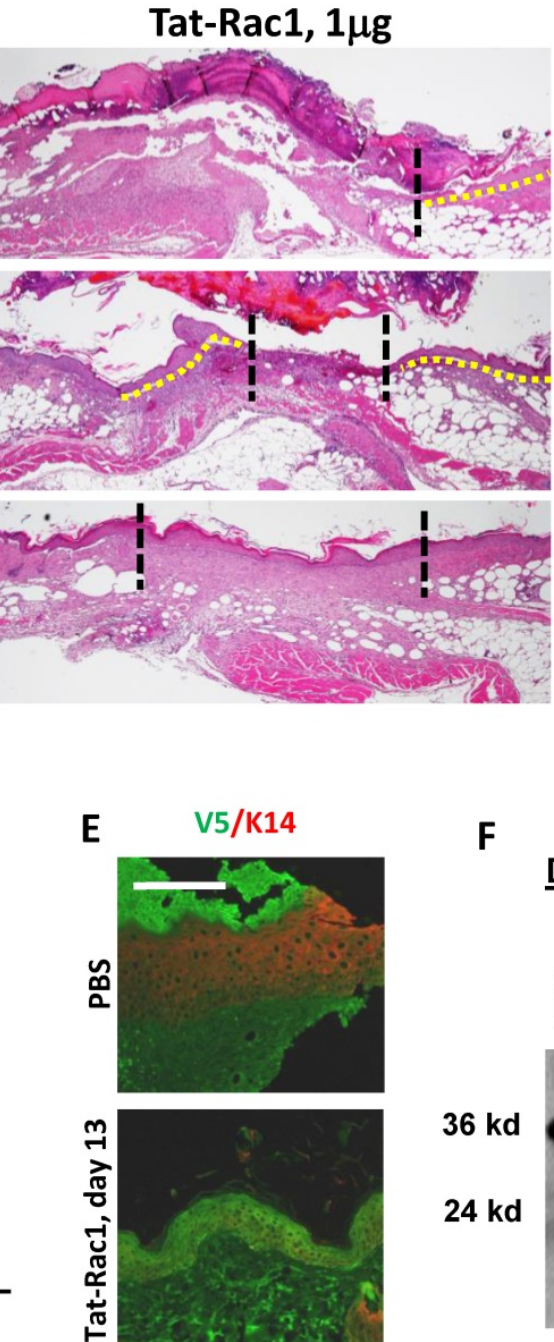

$\mathbf{F}$

Day 8

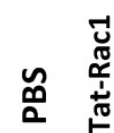

36 kd

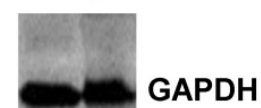

24 kd

V5

Figure 3. Topical application of Tat-Racl protein accelerated cutaneous wound closure in db/db mice. (A) Macroscopic appearance of wound closure in Tat-Racl and PBS treated db/db mice at different time points after wounding. Day 0 shows the size of 6-mm excisional wounds. (B) Quantification of wound area in Tat-Racl and PBS treated db/db wounds. The data represent 12 skin wounds per time point in each group (mean $+\mathrm{SD})$. (C) Representative H\&E wound sections from Tat-Racl (1 $\mu \mathrm{g} /$ wound) and PBS treated wounds on day 6, 8 and 13 after wounding. Dotted black lines delineate wound edge. Dotted yellow lines highlight re-epithelialized migrating tongues. Scale bar $=200 \mu \mathrm{m}$ for all sections. (D) Quantification of remaining wound widths on H\&E sections at different time points in Tat-Racl and PBS treated wounds during wound healing. Three skin wounds per time point from each group were analyzed. (E) Immunofluorescence staining of V5 labeling cell (green) in day 13 wounded skin treated by Tat-Racl or PBS control. K14 (red) was used as a counterstain for the epidermis. Hazy green (lower left) in PBS panel represents non-specific staining. Scale bar=100 $\mu m$ for both panels. (F) Tat-Racl western blot using a V5 antibody on protein extracted from day 8 wounds. GAPDH is the loading control. 
A

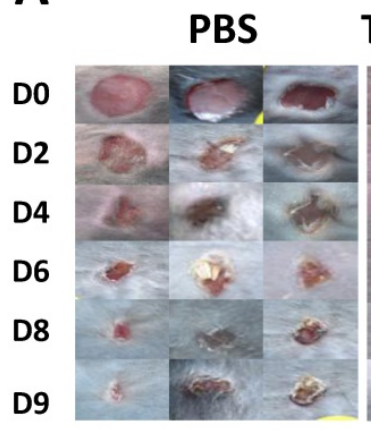

Tat-Rac1, $1 \mu \mathrm{g}$

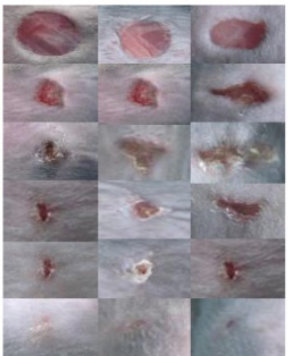

B

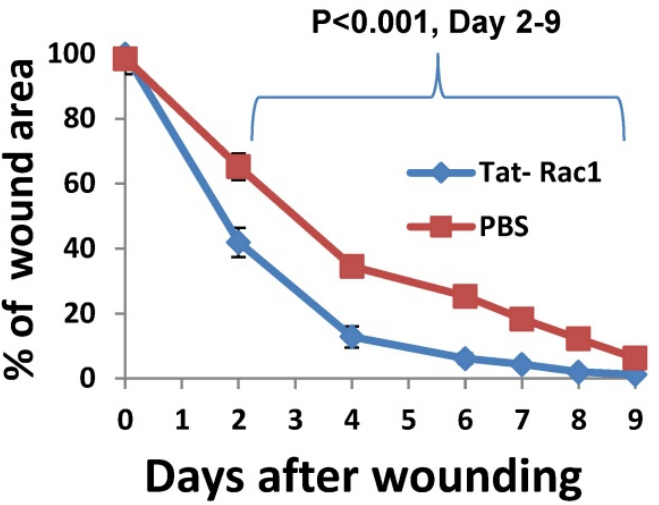

Tat-Rac1, $1 \mu \mathrm{g}$

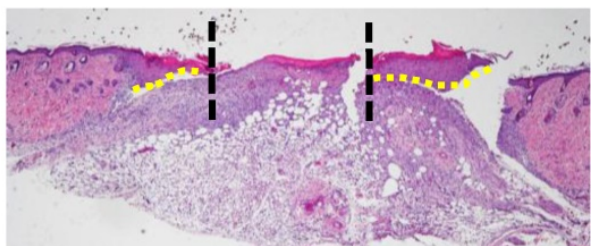

Scale bar: $200 \mu \mathrm{m}$
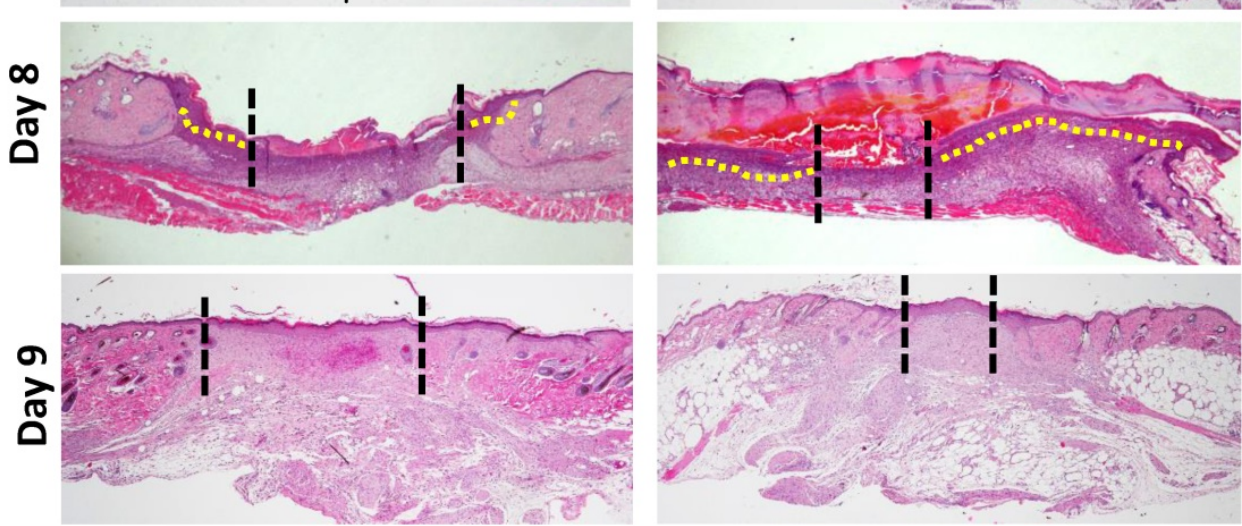

D

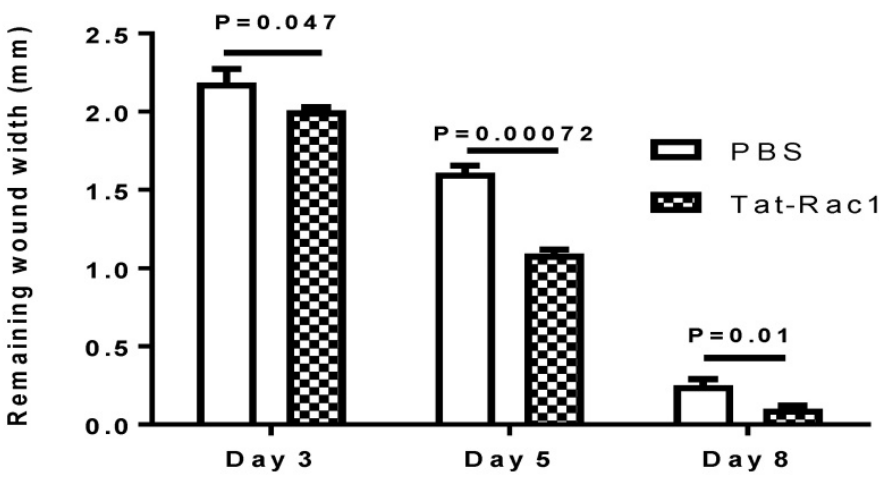

Figure 4. Topical application of Tat-Racl protein accelerated cutaneous wound closure in WT mice. (A) Macroscopic appearance of wound closure in Tat-Racl $(1 \mu \mathrm{g} /$ wound) and PBS treated WT wounds at different time points after wounding. Day 0 shows the size of a 6-mm excisional wound. (B) Quantification of wound area at different time points in Tat-Racl and PBS treated WT wounds after wounding. The data represents analysis of 12 skin wounds at each time point per group (mean + SD). (C) Representative H\&E sections in Tat-Racl and PBS treated WT wounds. Dotted black lines delineate wound edge. Dotted yellow lines highlight re-epithelialized migrating tongue. Scale bar: $200 \mu \mathrm{m}$ for all panels. (D) Quantification of remaining wound widths on H\&E sections at different time points in Tat-Racl and PBS treated wounds during wound healing. Three skin wounds per time point from each group were included for analysis. 


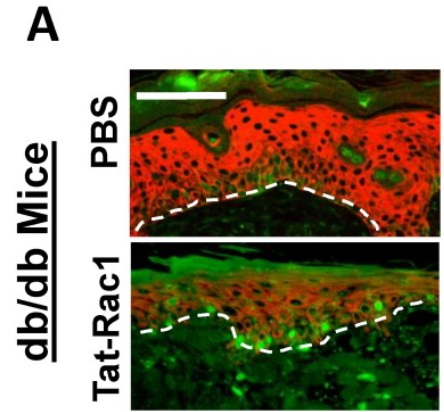

Day 6
BrdU/K14

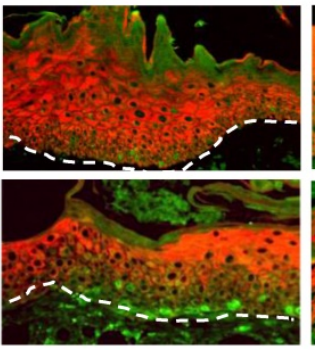

Day 8

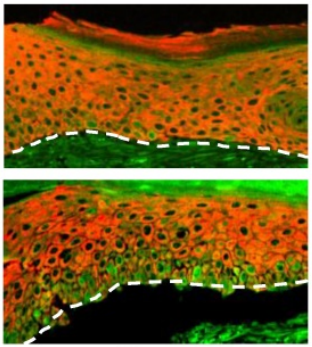

Day 13
B

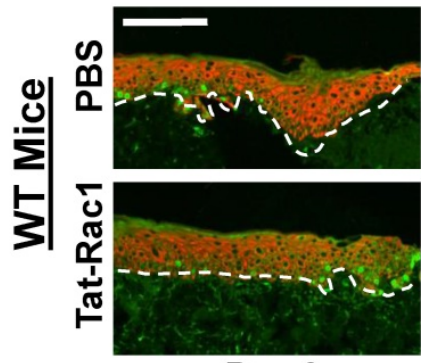

Day 3

\section{BrdU/K14}

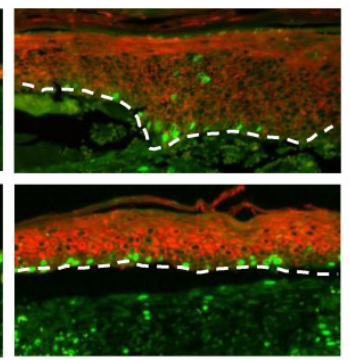

Day 5

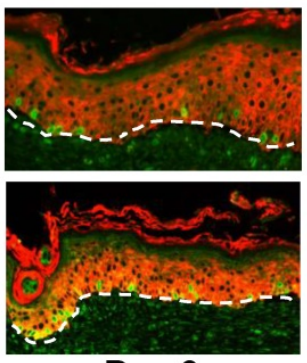

Day 9
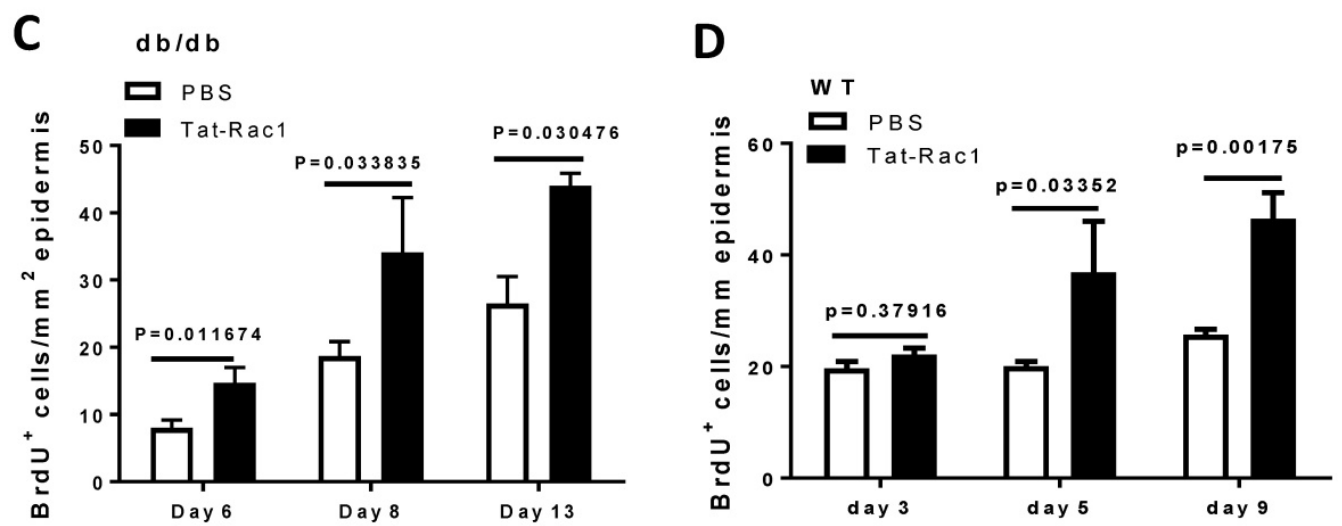

Figure 5. Increased keratinocyte proliferation in Tat-Racl-treated wounds. (A) and (B) Representative images of BrdU nuclear staining (green) in Tat-Racl and PBS treated db/db or WT wounds at different time points after wounding. Antibody against K14 (red) was used to counterstain the epidermis. Scale bar=50 $4 m$ for all images. Dotted lines indicate the boundary of the epidermis and the dermis. (C) and (D) Quantification of BrdU+ cells in db/db or WT wounds. The data represent mean \pm SD from analysis of three wounds per time point from each group.

\section{Discussion}

In the current study, we found that wounds in $\mathrm{db} / \mathrm{db}$ mice had a delayed increase in Rac1 protein and lower levels compared to wounds in WT mice. This defect was partially overcome by delivery of exogenous Tat-Rac1 protein to wounds in $\mathrm{db} / \mathrm{db}$ mice. This result is consistent with our previous study indicating that increased total Rac1 protein results in Rac1 activation that promotes healing of oral mucositis lesions [12].

Similar to our findings, a previous study reported that intravitreal injection of a constitutively active Tat-Rac1 protein for two weeks improved retina ganglion cell survival and regeneration [13]. Because constitutively active Rac1 is commonly expressed in human cancer [14] and treating diabetic wounds in patients could require extended treatment regimens, it is critical to avoid potential oncogenic effects from Rac1, as Rac1 activation is an important mechanism for cancer cell migration and metastasis [15]. Another consideration is the amount of Tat-Rac1 to use in wound healing, as cancer cells express much higher levels of Rac1 than normal cells [16, 17]. For these reasons, we did not use constitutively active Rac1, and also used a relatively low amount of Tat-Rac1 to treat wounds in vivo. The combination of using normal (not constitutively active) Rac1 with a 
low dose could attribute to a relatively modest effect of Rac1 on $\mathrm{db} / \mathrm{db}$ wounds. However, the same dose of Tat-Rac1 had more profound effects promoting healing in WT wounds than in $\mathrm{db} / \mathrm{db}$ wounds (Figure 3 vs. Figure 4). It is possible that although the Tat-Rac1-mediated epidermal proliferation, migration and reduced inflammation found in our study contributes to healing in both $\mathrm{db} / \mathrm{db}$ and WT wounds,

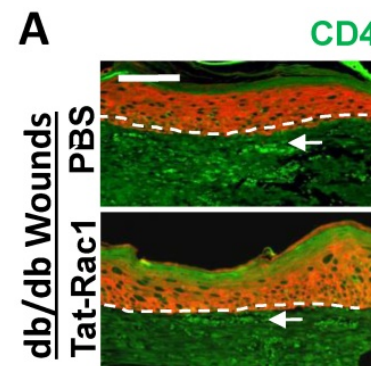

Day 6
CD45/K14

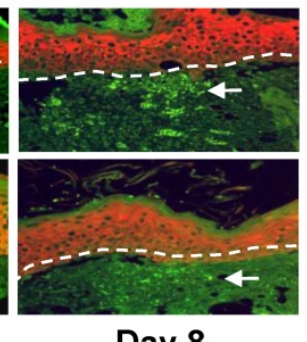

Day 8

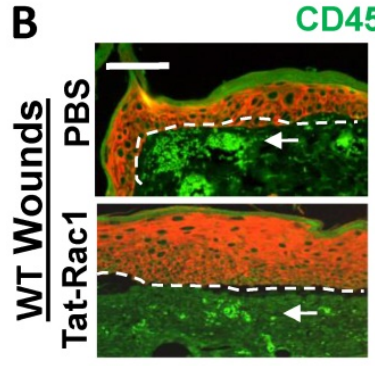

Day 3

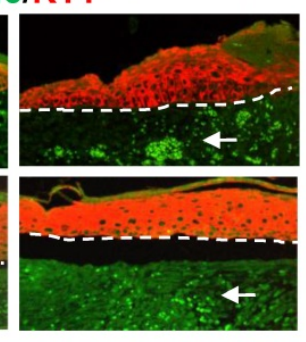

Day 5 the profound defects in these processes in $\mathrm{db} / \mathrm{db}$ wounds compared to WT wounds require a higher therapeutic dose or a more active form of Tat-Rac1. It is also probable that some of the defects in $\mathrm{db} / \mathrm{db}$ wound healing are not Rac1 targets thus cannot be corrected by Rac1 activation alone. These possibilities require future studies to identify the underlying mechanisms.

\section{C}

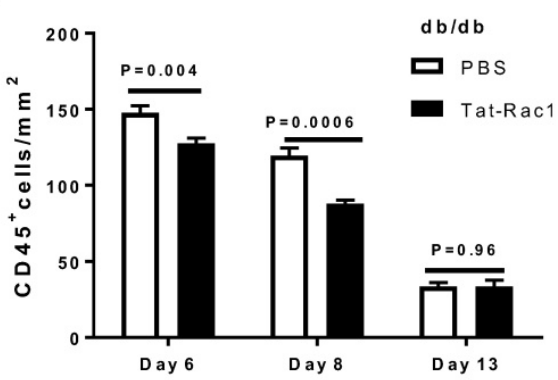

D

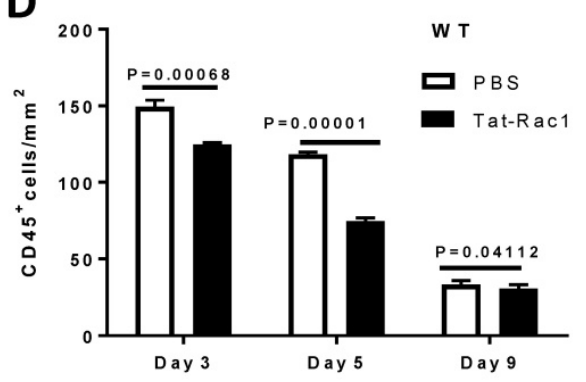

E

Day 8

Day 13

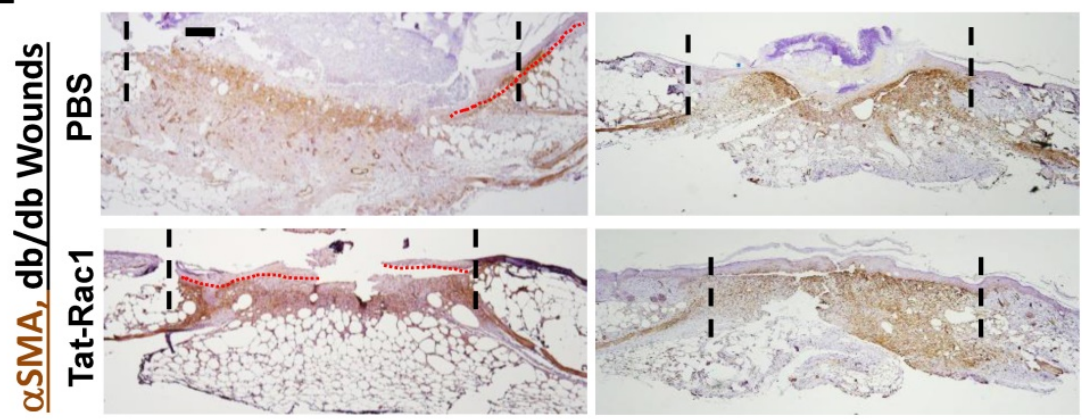

$\mathbf{F}$

Day 5

Day 9

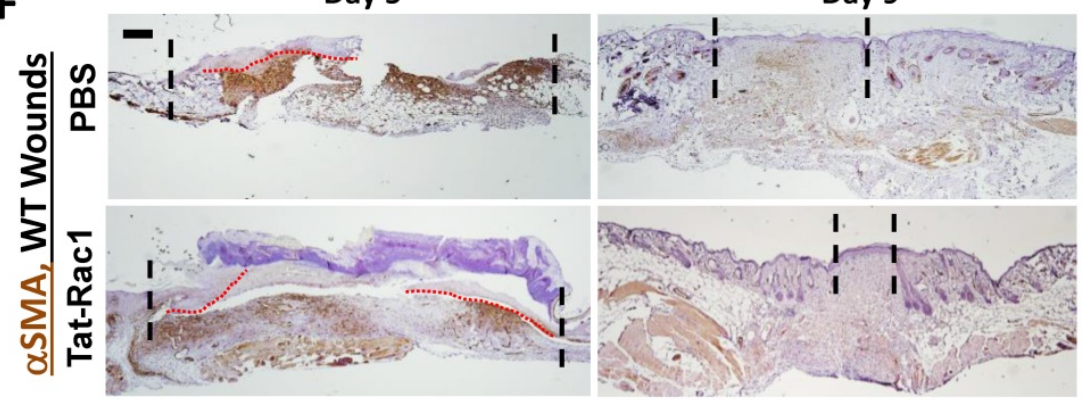

Figure 6. Reduced CD45+ cell numbers in Tat-Racl-treated wounds. (A) and (B) Representative images of CD45 staining in Tat-Racl and PBS treated db/db or WT wounds. Antibody against K14 (red) was used to counterstain the epidermis. Dotted lines indicate the boundary of the epidermis and the dermis. Scale bar represents $=50 \mu m$ for all images. Arrows point to examples of CD45+ cells (bright green). (C) and (D) Quantification of CD45 positive cells of db/db or WT wounds. The data represent mean \pm SD from analysis of three wounds from each time point. (E) and (F): IHC for aSMA staining. Scale bar: $400 \mu \mathrm{m}$ for all panels. Remaining granulation tissue is highlighted between the two dotted lines in each panel, red dotted lines delineate migrating epidermis. 
The effect of Tat-Rac1 on accelerating wound healing in normal mice is a bit surprising to us, as endogenous Rac1 is already elevated in response to wounding (Figure 1). Rac1 has been reported to promote proliferation in both keratinocytes and fibroblasts $[4-6,10]$ and our in vitro studies also show that Tat-Rac1 promoted proliferation and migration in both cell types (Figure 2). Because wound contraction contributes to wound closure in our wound healing model [18], it is important to determine if Tat-Rac1 promotes wound closure through keratinocytemediated re-epithelialization or/and fibroblast activation-induced wound contraction. We found that the in vivo effect of Tat-Rac1 was primarily in the wounded epidermis without early onset myofibroblast activation in granulation tissue of wounded skin (Figure 6F). Since Tat-Rac1 transduced both epidermal and dermal cells in vivo (Figure 3), these results suggest that the role of Rac1 in fibroblast activation is largely dispensable by other stimuli in the wound milieu. For example, transforming growth factor- $\beta$ (TGF $\beta$ ), a potent fibroblast activator, is elevated in wounds [19]. Further, $\alpha \mathrm{SMA}^{+}$fibroblasts did not linger after wound remodeling in Tat-Rac1 treated WT wounds (Figure 6F), suggesting that once Tat-Rac1 topical application is stopped, there is no sustained effect on fibroblast activation. Our data prompt future studies using models systems that more closely mimic acute surgical wounds and diabetic wounds in humans $[18,20]$ to further determine the effects of Tat-Rac1topical application on acute and chronic wounds.

Prolonged inflammation is one of the hallmark characteristics of chronic wounds [21]. We found that Tat-Rac1 treated wounds had reduced leukocyte infiltration prior to complete wound closure, suggesting that reduced inflammation by Tat-Rac1 could contribute to accelerated wound closure. Previous studies regarding the role of Rac1 in skin inflammation are controversial. Pedersen et al. have found that keratinocyte-restricted deletion of Rac1 promoted skin inflammation in an contact dermatitis model [22]. In contrast, Winge et al. showed that constitutive Rac1 is consistently elevated in psoriatic epidermis and that transgenic mice expressing a constitutively active form of Rac1 in the epidermis developed lesions similar to those in human psoriasis with aberrant activation of immune cells [23]. These paradoxical results from different model systems indicate that the effect of Rac1 on inflammation could depend on the amount of Rac1 protein as well as the disease context. In combination, these factors differentially affect Rac1 in regulating interactions between keratinocyte and immune cell.
In summary, our study revealed delayed and low levels of Rac1 expression in response to wounding in $\mathrm{db} / \mathrm{db}$ mice. Our study demonstrates the feasibility of topical, short-term application of a Rac1-based biologic to promote wound healing in both normal and diabetic wounds. Future studies will assess dose-dependent efficacy of Tat-Rac1 as a therapeutic agent and potential side effects, particularly oncogenic effects as a consequence of treatment.

\section{Abbreviations}

Rac1: Rac family small GTPase 1; Tat: transactivator of transcription; PDGF: platelet derived growth factor; BrdU: bromodeoxyuridine; WT: wild type; PBS: phosphate buffer saline.

\section{Acknowledgements}

The authors thank Haibin $\mathrm{Wu}$ for technical assistance, and Pamela Garl for critical proof reading of this manuscript. The work was supported by NIH R01 AR61792 to XJW. XJW is also a Research Biologist in Department of Veterans Affairs. TW is supported by the National Natural Science Foundation of China (No. 81372061). ZJ was a visiting scholar supported by the National Natural Science Foundation of China (No. 81402599). GH is currently supported by the National Natural Science Foundation of China (81472903 and 81673079). The contents do not represent the views of the U.S. Department of Veterans Affairs or the United States Government.

\section{Competing Interests}

The authors have declared that no competing interest exists.

\section{References}

1. Sen CK, Gordillo GM, Roy S, Kirsner R, Lambert L, Hunt TK, et al. Human skin wounds: a major and snowballing threat to public health and the economy. Wound repair and regeneration : official publication of the Wound Healing Society [and] the European Tissue Repair Society. 2009; 17: 763-71.

2. Eming SA, Martin P, Tomic-Canic M. Wound repair and regeneration: Mechanisms, signaling, and translation. Sci Transl Med. 2014; 6.

3. Werner S, Grose R. Regulation of wound healing by growth factors and cytokines. Physiol Rev. 2003; 83: 835-70.

4. Castilho RM, Squarize $\mathrm{CH}$, Leelahavanichkul K, Zheng $\mathrm{Y}$, Bugge $\mathrm{T}$, Gutkind JS. Rac1 is required for epithelial stem cell function during dermal and oral mucosal wound healing but not for tissue homeostasis in mice. PloS one. 2010; 5: e10503.

5. Tscharntke M, Pofahl R, Chrostek-Grashoff A, Smyth N, Niessen C, Niemann $\mathrm{C}$, et al. Impaired epidermal wound healing in vivo upon inhibition or deletion of Rac1. J Cell Sci. 2007; 120: 1480-90.

6. Liu SX, Kapoor M, Leask A. Rac1 Expression by Fibroblasts Is Required for Tissue Repair in Vivo. American Journal of Pathology. 2009; 174: 1847-56.

7. Hassanain HH, Irshaid F, Wisel S, Sheridan J, Michler RE, Goldschmidt-Clermont PJ. Smooth muscle cell expression of a constitutive active form of human Rac 1 accelerates cutaneous wound repair. Surgery. 2005; 137: 92-101.

8. Dam DHM, Wang XQ, Sheu S, Vijay M, Shipp D, Miller L, et al. Ganglioside GM3 Mediates Glucose-Induced Suppression of IGF-1 Receptor-Rac1 Activation to Inhibit Keratinocyte Motility. Journal of Investigative Dermatology. 2017; 137: 440-8.

9. Wang XQ, Lee S, Wilson H, Seeger M, Iordanov H, Gatla N, et al. Ganglioside GM3 Depletion Reverses Impaired Wound Healing in Diabetic Mice by 
Activating IGF-1 and Insulin Receptors. Journal of Investigative Dermatology. 2014; 134: 1446-55.

10. Roper JA, Williamson RC, Bally B, Cowell CAM, Brooks R, Stephens P, et al. Ultrasonic Stimulation of Mouse Skin Reverses the Healing Delays in Diabetes and Aging by Activation of Rac1. Journal of Investigative Dermatology. 2015; 135: 2842-51.

11. Han G, Li F, Ten Dijke P, Wang XJ. Temporal smad7 transgene induction in mouse epidermis accelerates skin wound healing. Am J Pathol. 2011; 179: 1768-79.

12. Han G, Bian L, Li F, Cotrim A, Wang D, Lu J, et al. Preventive and therapeutic effects of Smad7 on radiation-induced oral mucositis. Nat Med. 2013; 19: 421-8.

13. Lorenzetto E, Ettorre M, Pontelli V, Bolomini-Vittori M, Bolognin S, Zorzan S, et al. Rac1 Selective Activation Improves Retina Ganglion Cell Survival and Regeneration. PLoS One. 2013; 8.

14. Singh A, Karnoub AE, Palmby TR, Lengyel E, Sondek J, Der CJ. Rac1b, a tumor associated, constitutively active Rac1 splice variant, promotes cellular transformation. Oncogene. 2004; 23: 9369-80.

15. Kazanietz MG, Caloca MJ. The Rac GTPase in Cancer: From Old Concepts to New Paradigms. Cancer Res. 2017; 77: 5445-51.

16. Ungefroren $\mathrm{H}$, Witte $\mathrm{D}$, Lehnert $\mathrm{H}$. The role of small GTPases of the Rho/Rac family in TGF-beta-induced EMT and cell motility in cancer. Developmental dynamics : an official publication of the American Association of Anatomists. 2018; 247: 451-61.

17. Crnogorac-Jurcevic T, Efthimiou E, Capelli P, Blaveri E, Baron A, Terris B, et al. Gene expression profiles of pancreatic cancer and stromal desmoplasia. Oncogene. 2001; 20: 7437-46.

18. Bhatia A, O'Brien K, Guo J, Lincoln V, Kajiwara C, Chen M, et al. Extracellular and Non-Chaperone Function of Heat Shock Protein-90alpha Is Required for Skin Wound Healing. J Invest Dermatol. 2018; 138: 423-33.

19. Wang XJ, Han G, Owens P, Siddiqui Y, Li AG. Role of TGF beta-mediated inflammation in cutaneous wound healing. J Investig Dermatol Symp Proc. 2006; 11: 112-7.

20. O'Brien K, Bhatia A, Tsen F, Chen M, Wong AK, Woodley DT, et al. Identification of the critical therapeutic entity in secreted Hsp90alpha that promotes wound healing in newly re-standardized healthy and diabetic pig models. PLoS One. 2014; 9: e113956.

21. Eming SA, Martin P, Tomic-Canic M. Wound repair and regeneration: Mechanisms, signaling, and translation. Science translational medicine. 2014; 6: 265sr6.

22. Pedersen E, Wang ZP, Stanley A, Peyrollier K, Rosner LM, Werfel T, et al. RAC1 in keratinocytes regulates crosstalk to immune cells by Arp2/3-dependent control of STAT1. J Cell Sci. 2012; 125: 5379-90.

23. Winge MCC, Ohyama B, Dey CN, Boxer LM, Li W, Ehsani-Chimeh N, et al. RAC1 activation drives pathologic interactions between the epidermis and immune cells. Journal of Clinical Investigation. 2016; 126: 2661-77. 